measured, to determine which chemical fraction contains the atoms (for example, in the liquid chromatography experiments, whether the atoms come through the column or stick to it). These experiments have evolved from a few to several hundred manual operations, with the gradual addition of automation to increase both speed and reliability, until, in the very advanced miniature automated chemistry described in the present paper, even the chromatographic columns are changed automatically, and several thousand experiments are repeated.

To what elements can chemistry be extended? Older predictions were that chemical experiments would end in the heavy actinides. Using present-day production methods and available targets, it is not likely that chemical studies will extend beyond one or two more elements. Yet new nuclides with half-lives long enough for chemical studies are predicted, at least up to element 114 . Only a few atoms of these nuclides might be produced in months of bombardment. Intense radioactive beams using neutronrich projectiles are one possible route to increased production, still far in the future - but perhaps not so far, when we note that the first chemistry of seaborgium has come 23 years after its discovery.

Ron Lougheed is in the Lawrence Livermore

National Laboratory, University of California,

Livermore, California 94550, USA.

1. Schädel, M. Radiochim. Acta 70, 207-223 (1995).

2. Schädel, M. et al. Nature 388, 55-57 (1997).

3. Kratz, J. V. et al. Radiochim. Acta 48, 121-133 (1989).

4. Gregorich, K. E. et al. Radiochim. Acta 43, 223-231 (1988).

\title{
Colouring the cortex
}

\section{Karl R. Gegenfurtner}

I humans, the neural basis for colour vision lies in the activity of the 'colouropponent' neurons, which receive inputs of opposite sign from the three different classes of cone photoreceptors that are found in the eye (Fig. 1). Colour-opponent neurons are abundant in the first stages of the visual pathway - the retina and the lateral geniculate nucleus ${ }^{1}$. Surprisingly, however, they are observed rather infrequently by

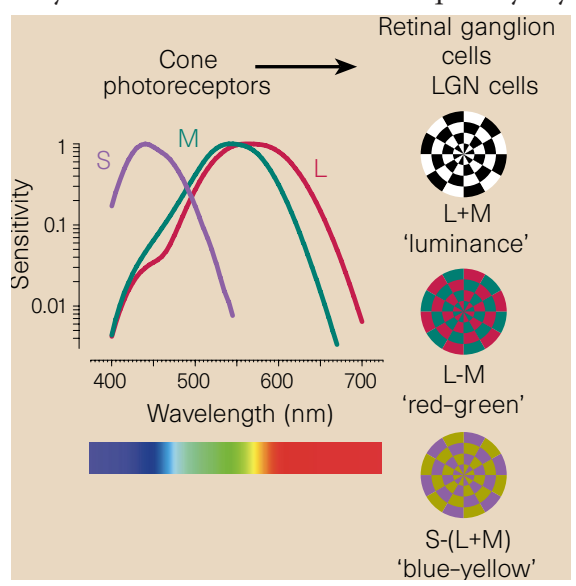

Figure 1 In human colour vision, the information from the three different types of cone photoreceptors, short-, middle- and longwavelength-sensitive $(S, M, L)$, is combined by retinal ganglion cells and cells in the lateral geniculate nucleus (LGN) into two chromatic channels and a luminance channel ${ }^{1}$. The spectral sensitivities of the cone photoreceptors are shown to the left. Chromatic channels receive signals of opposite sign from the cones. These opponent channels are often labelled 'red-green' and 'blue-yellow', even though the stimuli that differentially activate them (shown to the right) appear to be a noticeably different colour ${ }^{11}$. Increasing contrast results in the appearance of more saturated colours. single-cell recordings in the next stage, the primary visual cortex (V1), where neurons that add inputs from all three cone types predominate $^{2}$.

On page 68 of this issue, Engel et al. report that, in sharp contrast to the singlecell recordings, most of the activity that is detected by functional magnetic resonance imaging (fMRI) of visual cortical areas V1 and V2 in humans is due to the activity of colour-opponent cells. Until now, most fMRI studies have only shown that there is a change in activity in a defined brain region when a particular stimulus is used. But Engel et al. have measured differential responses to subtle changes in colour and contrast, so the levels of activity in the fMRI signal can be used to produce response contours for the whole range of colours. By comparing these responses with behavioural measurements obtained for the same stimuli and observers, they have found that the activity of the red-green and blue-yellow colour-opponent mechanisms seems to underlie both sets of data.

Less than ten years ago, in a survey of neuroscientific methods, Churchland and Sejnowski ${ }^{4}$ exposed a gaping hole in the research - namely, that no method existed to study the activity of large ensembles of neurons, or even whole cortical areas, over a time course of several seconds. The subsequent advances in imaging techniques, and especially the availability of fMRI (ref. 5), promised the missing link (Fig. 2).

Functional MRI is based on bloodoxygen-level-dependent (bold) changes in the magnetic resonance signal. The bold assumption is that the blood oxygen level is an indicator for local cortical activity. Because there is activity everywhere - even in the resting brain - only the activity changes that are induced by stimuli differing in the attribute of interest are measured. The resulting difference in the fMRI signal is typically about five per cent of the total signal, and it depends on the stimulus that is used. For example, if a region responds differentially to a moving stimulus, it is tagged as the 'motion centre' in the brain. Several such processing centres have been found, and the trend is going towards a 'grandmother centre'. This is analogous to the trend towards increasingly specialized processing units ${ }^{6}$, which came about from single-cell recordings during the 1970s. Clearly, it is implicitly assumed that information processing is managed by specialized brain regions distributed processing goes unnoticed in this standard approach.

More recently, several groups ${ }^{7,8}$ have developed strategies to distinguish between visual cortical areas by exploiting the system-

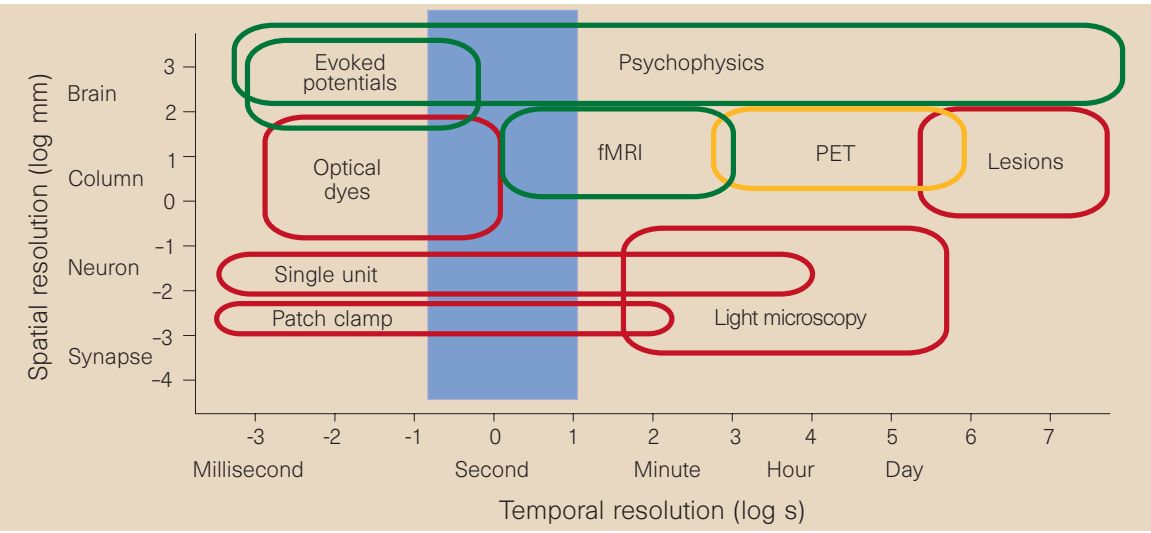

Figure 2 Spatial and temporal resolution of some of the most common experimental methods that are used to study sensory processing. Green boxes indicate non-invasive methods; red boxes indicate invasive methods. The blue shaded area indicates a time slice of major interest, between $100 \mathrm{~ms}$ and $10 \mathrm{~s}$. Engel et al. ${ }^{3}$ used functional magnetic resonance imaging (fMRI) to show that most of the activity in the visual cortical areas V1 and V2 in humans is due to the activity of colour-opponent cells. The precise resolution of fMRI is often overestimated - even though images can be acquired every $50 \mathrm{~ms}$ with an accuracy of $1 \mathrm{~mm}$, the underlying changes in blood oxygen level are much slower and coarser ${ }^{12}$. PET, positron emission tomography. (Modified from ref. 4.) 
atic differences in their visual-field representations. In this way, Engel et al. ${ }^{3}$ initially identified $\mathrm{V} 1$ and $\mathrm{V} 2$, and then determined the colour tuning of these two regions. They used flickering pinwheel stimuli of different contrast (Fig. 1), and measured the fMRI response averaged over the whole of $\mathrm{V} 1$ and V2 separately. They found that there was a systematic increase in the fMRI signal with increasing contrast in the stimulus. By repeating this for several different colour combinations, they worked out response contours of equal activity for these brain regions. The result was a map of the activity of two independently identified cortical areas - V1 and V2 - to any colour combination.

What singles out this study is that the fMRI data can be readily correlated with psychophysically obtained measurements. Indeed, the similarity between the psychophysical threshold measurements and the fMRI responses of the same observers to the same stimuli is quite stunning. In both experiments the best responses are obtained for coloured stimuli, the perception of which is mediated by colour-opponent processing. There are also remarkable differences. Most notably, whereas the fMRI data show a bigger response to colour than luminance throughout, psychophysically this holds only for a highly restricted range of stimuli, those with little spatial or temporal variation $^{9}$. Although the fMRI measurements are about 100 times less sensitive than the psychophysical measurements, if we assume that the contours can be directly related to one another, the implication is that there is substantial temporal filtering of the chromatic signal after the $\mathrm{V} 1$ region.

The fMRI response to coloured stimuli is also greater than would be expected based on single-cell recordings in Old World monkeys, whose cones and pathways for early visual processing are similar to those in humans. Here, fewer than 15 per cent of all cells give a larger response to colour than to luminance ${ }^{2}$. So why is the fMRI response dominated by the activity of colour-opponent cells instead of luminance cells? To answer this, we need to look at the relationship between single-unit activity and fMRI response. Does the fMRI signal simply reflect the average activity of all neurons? And how specific is the response of an individual neuron to a particular stimulus?

Many of the 'luminance' cells in the V1 region have highly complex receptive-field properties. Their responses are determined by many conjoined properties of the stimulus, including orientation, spatial frequen$c y$, temporal frequency, direction and size ${ }^{10}$. In single-unit recordings, the idea is to get all of these parameters right for the individual cell, so there will be a strong response to the optimal stimulus but little response to everything else. Because each cell gets its optimal stimulus, a number of highly active cells are observed. However, it is not clear how many of these cells respond to a particular stimulus, such as the pinwheel used by Engel and colleagues. There might even be an advantage in having as few neurons as possible respond to each luminance stimulus $^{6}$. But the goal could be quite different for colour. Here, the visual system might want to attach a colour to each stimulus, independently of its other characteristics. So it would make sense for colour cells to respond in a less specific manner to these other features.

Although other interpretations of some aspects of these data are possible, the exciting prospect is that we can now study the functional transformation of the response across different visual areas, and correlate the signals to single-cell responses in these areas as well as to psychophysical responses.

Karl R. Gegenfurtner is at the Max-Planck-Institut für biologische Kybernetik, Spemannstraße 38, 72076 Tübingen, Germany.

1. Derrington, A. et al. J. Physiol. (Lond.) 357, 241-265 (1984)

2. Lennie, P. et al. J. Neurosci. 10, 649-669 (1990).

3. Engel, S., Zhang, X. \& Wandell, B. Nature 388, 68-71 (1997).

4. Churchland, P. S. \& Sejnowski, T. J. Science 242, 741-745 (1988).

5. Kwong, K. K. et al. Proc. Natl Acad. Sci. USA 89, 5675-5679 (1992).

6. Barlow, H. in Cognitive Neuroscience (ed. Gazzaniga, M. S.) 415-435 (MIT, Cambridge, MA, 1995).

7. Sereno, M. I. et al. Science 268, 889-893 (1995).

8. DeYoe, E. et al. Proc. Natl Acad. Sci. USA 93, 2382-2386 (1996).

9. Kelly, D. H. J. Opt. Soc. Am. 73, 742-750 (1983).

10. Hubel, D. H. \& Wiesel, T. N. Proc. R. Soc. Lond. B 198, 1-59 (1977).

11. Krauskopf, J., Williams, D. R. \& Heeley, D. W. Vision Res. 22, 1123-1131 (1982).

12. Malonek, D. \& Grinvald, A. Science 272, 551-554 (1996).

\section{Not-so-cosmic rays}

\section{Peter L. Biermann}

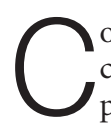

osmic rays are energetic particles that constantly hit the Earth. They include protons and heavier nuclei, along with a few electrons and positrons. It is usually assumed that the flux of cosmic rays is roughly the same throughout our Galaxy, but new data published by Anatolya Erlykin and Arnold Wolfendale may mean that what we see contains the signature of a nearby source, probably a young supernova remnant ${ }^{1}$

Cosmic rays have been seen up to an energy of nearly $10^{21}$ electron volts $(\mathrm{eV})$ - far beyond any dream of the physicists at the particle accelerators at Stanford, Fermilab or CERN. The flux is very low, from about 10 particles $\mathrm{cm}^{-2} \mathrm{~s}^{-1}$ at energies near $10^{9} \mathrm{eV}$, to about 1 particle $\mathrm{km}^{-2}$ per century near $10^{20} \mathrm{eV}$.

More than sixty years ago, Walter Baade and Fritz Zwicky pointed out ${ }^{2}$ that the beautiful nebulae left behind by exploding stars - such as the supernova of AD 1054 observed by Chinese astronomers - could be the powerhouses that accelerate cosmic rays. The standard theory, originally conceived in its basic form by Enrico Fermi about fifty years ago ${ }^{3,4}$, holds that cosmic rays gain their energy in shock waves created by the explosion of stars. Charged energetic particles gyrate around magnetic fields on both sides of a shock, and bounce off irregularities in these magnetic fields or off turbulence in the gas. Some of these particles bounce back and forth across the shock, gaining energy all the time because the two flows are moving towards each other - compression heats, as a bicycle pump demonstrates.

Three things limit the maximum energy that a particle can gain in this process. First, it can run out of time: the shock gets slower as it engulfs more material, and therefore less efficient at accelerating particles. Second, it can run out of room: a charged particle gyrating in a magnetic field needs some space for its helical motion, and this space increases with energy (and decreases with the charge on the nucleus), so at a certain energy the gyration can become bigger than the shock wave. Third, it can hit something

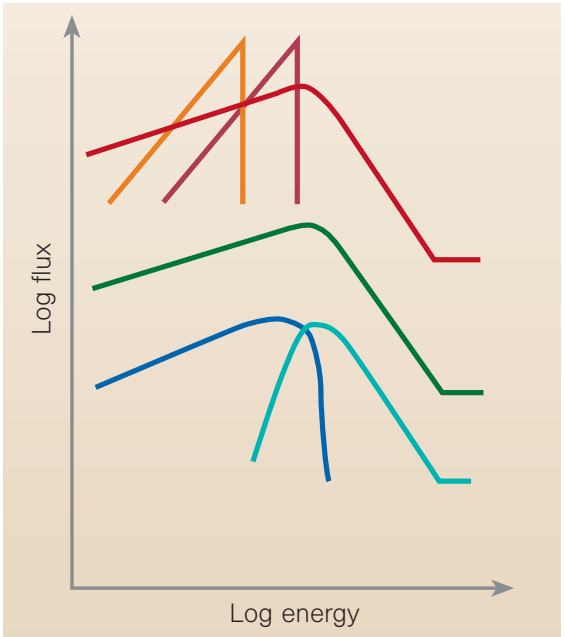

Figure 1 Three knees. The spectrum of cosmic rays hitting the Earth steepens at an energy of about $10^{15} \mathrm{eV}$ (at the ' $\mathrm{knee}$ '), and it is relatively easy to explain their production up to about this energy. That could mean: a, that there are two different types of source, which have spectra that join at the knee; or b, that the lower-energy cosmic rays somehow get accelerated, inefficiently, to energies above the knee. But now there is also evidence for bumps, or 'teeth', below the knee, which could be due to different elements accelerated in a nearby supernova remnant. That would mean some changes to either a or b, such as for instance shown in c. 